\title{
Study of the effects of human tissue on performance of a loop antenna
}

\author{
Khaoula Tayari ${ }^{1}$, Houda Werfelli ${ }^{2}$, Mondher Chaoui ${ }^{3}$, Hamadi Ghariani ${ }^{4}$, \\ Mongi Lahiani ${ }^{5}$ \\ Department of Electrical Engineering, LETI laboratory, National School of Engineering of Sfax, University of
} Sfax, Tunisia

\begin{abstract}
In this paper, the issue of interactions of electromagnetic field with biological tissues is asked. Its interactions essentially depend on the electrical properties of the medium, themselves dependent on the frequency. The body is a loss dielectric medium. It interacts with the near field of the antenna; it will cause changing on antenna characteristics, reducing in performance and changing in impedance. This study of interaction is made with a rectangular loop antenna placed near a simplified model of the human body on ISM band especially $433 \mathrm{MHz}$.
\end{abstract}

Keywords: Depth of penetration, electromagnetic field, human body, interaction human-antenna, wavelength

\section{Introduction}

Communication networks have become indispensable in our daily lives. So it will be necessary to highlight the influence of the body on the characteristics of the antenna. Indeed, the diversity and quantity of electronic devices used in daily life, requires a thorough understanding of the propagation of electromagnetic waves through the human body. In addition, understanding of the current flow through the human tissue has the greatest interest in the analysis of several applications in the medical field such as electrical stimulation, the diagnosis and treatment of various physiological actions with weak electric currents. In general, the electromagnetic field passing through the human body depends on the thickness and the exact composition of biological tissues. The human body is a multilayer medium (Fig.1). Each layer has its own dielectric characteristics. These properties were the interest of many researchers for over a century for many reasons. To characterize the response of a tissue to electromagnetic waves, the knowledge of the permittivity $\varepsilon_{\mathrm{r}}$ and the conductivity $\sigma$ is required. Main characteristics of the dielectric properties are: significant variations in conductivity and permittivity of around some frequency values due to dielectric relaxation phenomena, very large permittivity values at lower frequencies and large differences in properties between the tissues [1].

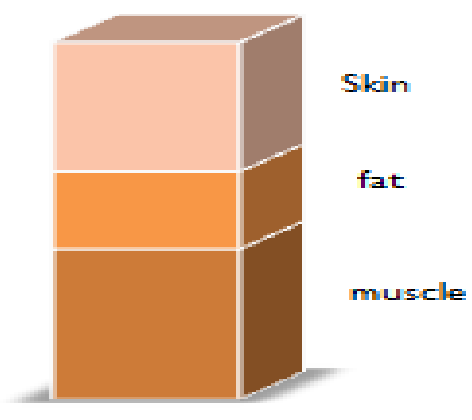

Fig.1. Model of human tissue

The human body does not present an ideal environment for the propagation of RF waves, especially for high frequencies. This dissipative environment will reduce RF antenna performance especially in terms of efficiency and radiation pattern; a large part of the power will be absorbed by the human body. The absorption due to human tissue is a problem in the design of antennas placed in the human body proximity. To achieve better performance with medical circuits, the type of antenna is one of the most important factors. Its shape can help to integrate antenna easily in the medical circuit. It has been shown that the loop antennas are more effective in equipment that is near the human body [2] [3] [4]. They have the same desirable characteristics as dipoles and monopoles in that they are inexpensive and simple to build. For this, our study will be made with this type of antenna. Therefore, in this paper, we will make a projection on the effect of electromagnetic waves on the biological tissue and on the effect of human body on the characteristics of a loop antenna, so a study of body-antenna interaction. 


\section{Theoretical Approach Of Tissue-Wave Interactions}

Biological medium are between dielectric (insulating) and perfect conductors. Mathematical modeling of the interaction of electromagnetic fields requires knowledge of the properties of the radiation source, the geometry of the body exposed as well as its electrical properties that are conductivity, permittivity and permeability which is equal to that of vacuum for biological tissues as they are considered non-magnetic. The electrical conductivity and the electrical permittivity are specific to each tissue and depend on many factors such as the frequency and the temperature.

The behavior of the electromagnetic field produced by an external source and propagating towards a biological medium can be described by the Maxwell equations using simple geometric configurations.

The wave vector is equal to:

$\underline{\mathrm{k}}=\frac{\omega}{\mathrm{c}}(\mathrm{n}+\mathrm{j} \kappa)=\beta+\mathrm{j} \alpha$

Where: $\mathrm{n}$ and $\kappa$ represent successively the real part and the imaginary part of the complex index $\underline{n}$ of a medium, such as:

$\underline{\mathrm{n}}=(\mathrm{n}+\mathrm{j} \kappa)=\sqrt{\varepsilon_{\mathrm{r}}\left(1-\frac{\mathrm{j \sigma} \sigma}{\omega \varepsilon}\right)}$

Where $\mathrm{n}$ is the refractive index characterizing the propagation of the wave and $\kappa$ represents the extinction index that characterizes the damping of the wave in the direction of propagation due to energy losses in the medium. While: $\alpha=\kappa \frac{\omega}{c}$ is the linear attenuation $(\mathrm{Np} / \mathrm{m})$ and $\beta=\mathrm{n} \frac{\omega}{\mathrm{c}}$ is the linear phase shift $(\mathrm{rad} / \mathrm{m})$.

Phase and group velocities are dependent on $\beta$ while the signal attenuation in the medium considered depends on $\alpha$. The values of $\alpha$ and $\beta$ are calculated from the complex expression of the wave vector and become:

$\alpha=\omega \sqrt{\frac{\varepsilon \mu_{0}}{2}\left[\sqrt{1+\frac{\sigma^{2}}{\varepsilon^{2} \omega^{2}}}-1\right]}$

$\beta=\omega \sqrt{\frac{\varepsilon \mu_{0}}{2}\left[\sqrt{1+\frac{\sigma^{2}}{\varepsilon^{2} \omega^{2}}}+1\right]}$

So, the wavelength of signal in the medium is given by:

$\lambda=\frac{2 \pi}{\beta}=\frac{\lambda_{0}}{\sqrt{\frac{\varepsilon \mathrm{r}}{2}\left[\sqrt{1+\frac{\sigma^{2}}{\varepsilon^{2} \omega^{2}}}+1\right]}}$

With: $\lambda_{0}=\frac{\mathrm{c}}{\mathrm{f}}$

The curve of Fig. 2 shows the changes in the wavelength in the muscle as a function of frequency. It shows that for a frequency of $10 \mathrm{MHz}$, the wavelength which propagates in the muscle is much lowering (divided by a factor equal to75) relative to the length of the wave in the air. The electrical parameters used to construct this figure (and the following) are extracted from [1].

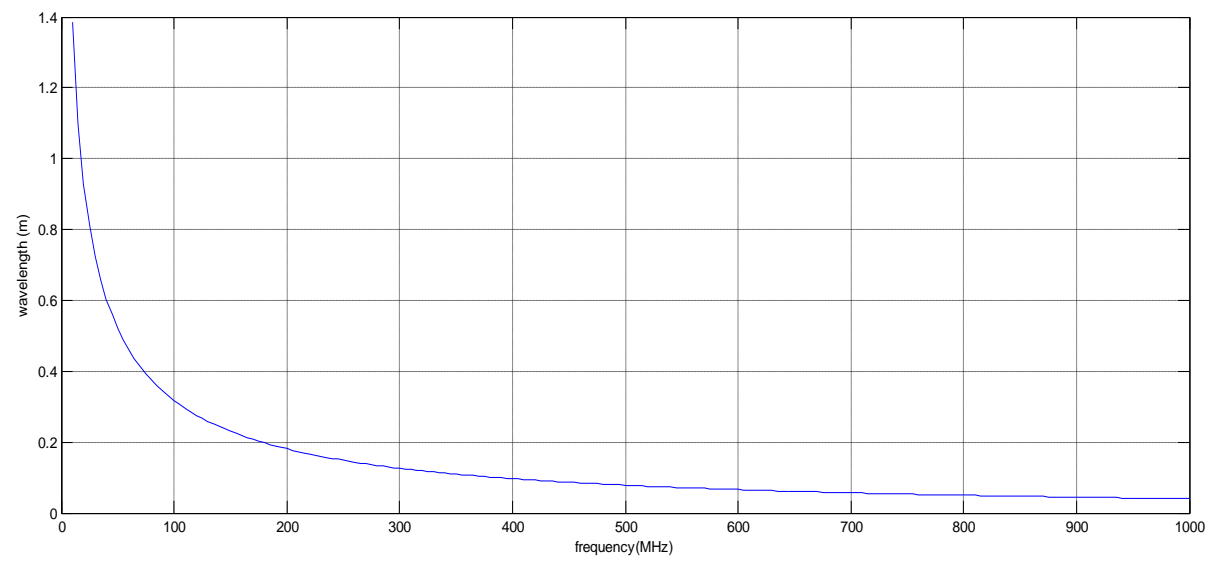

Fig.2. Variation of the wavelength in the muscle as a function of frequency 
The penetration of electromagnetic fields in the tissues will be limited very quickly because of the skin effect and the dissipation of energy in the medium. The skin effect results in a decrease of the field $\mathrm{E}(\mathrm{z})$ at a distance $\mathrm{z}$ from the interface according to the relation: $\mathrm{E}(\mathrm{z})=\mathrm{E}_{0} \mathrm{e}^{-\alpha \mathrm{z}}$ with $\mathrm{E}_{0}$ is the amplitude of the field at the interface level.

The depth of penetration (or skin depth) corresponds to the distance at the end of which the amplitude of the wave is reduced to $1 / \mathrm{e}$ of its initial value, whether:

$\delta=\frac{1}{\alpha}=\frac{1}{\omega \sqrt{\frac{\varepsilon \mu_{0}}{2}\left[\sqrt{1+\frac{\sigma^{2}}{\varepsilon^{2} \omega^{2}}}-1\right]}}$

Fig. 3 drawn from equation 6 shows that the skin effect is not prominent in low frequency and the depth of penetration decreases with frequency. For muscle, it is about $14 \mathrm{~cm}$ at $10 \mathrm{MHz}$ to reach $5 \mathrm{~cm}$ at $1 \mathrm{GHz}$. It decreases to values below the $\mathrm{mm}$ to greater than $25 \mathrm{GHz}$ frequencies. The higher the frequency of the signal, the higher the internal organs are protected from the effect of radiation through the outer layers of the human body.

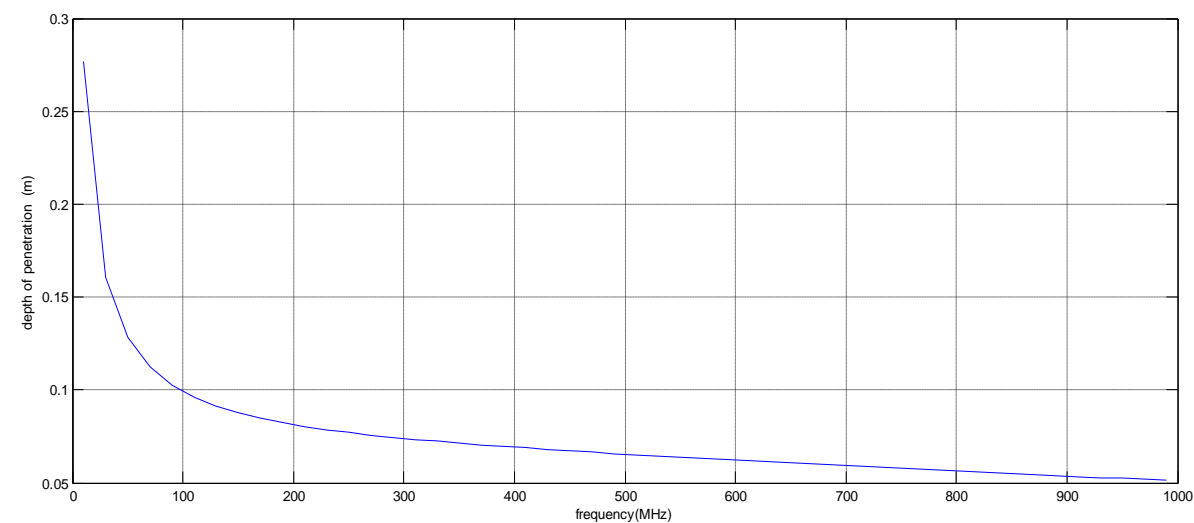

Fig.3. Changes in skin depth $\delta$ as a function of frequency for the muscle

\section{Body-antenna interaction}

The idea is to facilitate communication on the body wirelessly and give the user the maximum of comfort. Connectivity is achieved through antenna instead of wire. Ban system (Body Area Network), in general, is divided into various nodes that are processed by each antenna. When the antennas used for applications on the body, connectivity should have the following characteristics: Low Profile, compliant (easy to wear and to be powered) and body insulation with better efficiency on the body. Antenna design for BAN applications is a difficult task because the antenna must be small, efficient, and must not be influenced by the body of the user, what makes the isolation of the antenna of importance.

The study of the influence of the body on the operation of the antenna has been the subject of several works for forty years. In the first study published on the subject in 1968, Krupka presents an experimental study on the effect of the body when an operator holds in his hand a small transmitter operating between 30 and 150 $\mathrm{MHz}$ [5]. He concluded that the body acts as an absorbent element, reducing the radiated power when the antenna is close to the body. The author assumes that the body can be modeled by equivalent impedance consisting of a radiation resistance, loss resistance and reactance which depend on frequency. It shows that the body may have resonances, as an antenna, and the proximity of the two possibly give rise to a coupling resulting in the change in impedance, radiation pattern and a weaker gain. Thus, Krupka demonstrates experimentally that at low frequencies, the body acts as a director and that for higher frequencies (above $60 \mathrm{MHz}$ ); the body acts as a reflector. In 1972, an experimental study on the human body impedance shows that the body behaves like a dielectric cylinder losses [6]. In the 70 appear in the United States the first antennas on-body specifically dedicated to police [7] [8]. This multi-turn loops operating in VHF and placed on the operator's shoulder. A ground plane is associated with antennas with minimum dimensions required to prevent significant degradation of the antenna performance. In the first theoretical treatment of body-antenna interaction limited in V/UHF domain, the body is treated as a radiating element fed by coupling [9]. The interaction of the field emitted by the antenna and those re-radiated by the body are used to approximate the change in the impedance and the radiation pattern. It is also shown that for a $150 \mathrm{MHz}$ frequency, the body goes from director to reflector system for a height of $1 \mathrm{~m}$ or $\lambda / 2$. In 1980 published a study on the impedance of a dipole in and above the body [10]. King reuses a theory developed in the case of antennas very close to the soil in order to apply to the body and in particular to the case of a dipole to $3 \mathrm{GHz}$ [7]. To study the variations in impedance and efficiency of a dipole 
above the body, several researchers using equations developed in [11]. After the 80s, studies are mainly based on FDTD simulations and no analytical model is developed.

\section{Description of the body-antenna interaction phenomenon}

Fig. 4 summarizes the antenna body interactions. Thus, if the distance to the body is such that the reactive field zone penetrates inside thereof, there will be a gradual decrease in the yield and the antenna impedance undergoes significant changes.

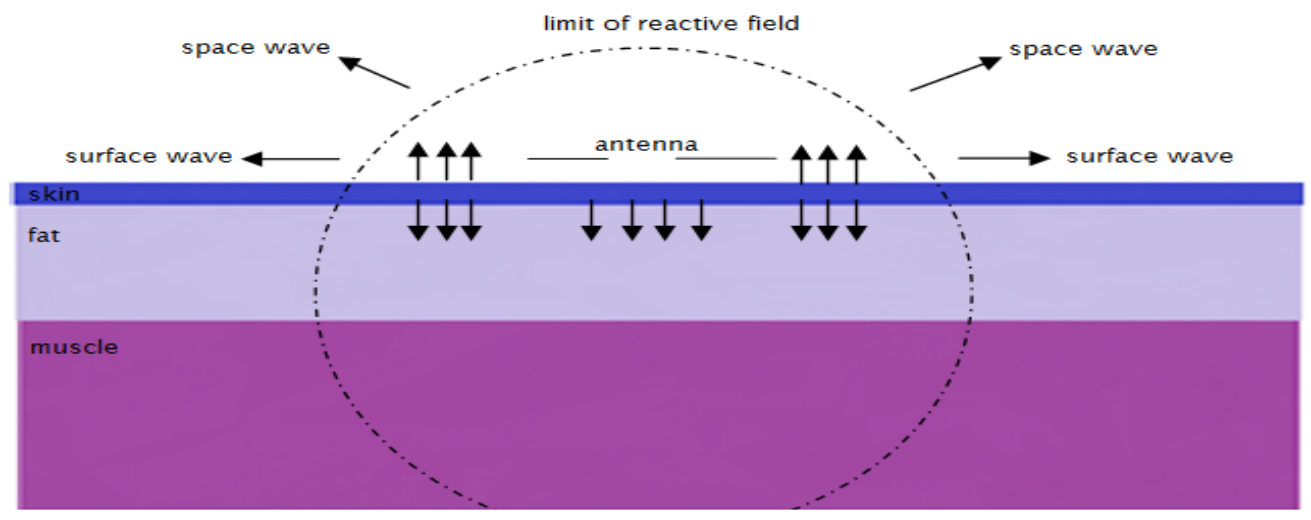

Fig.4.The mechanisms of body-antenna interaction

Next the fat thicknesses, it can also create standing waves between the skin and muscle leading to increased heat dissipation in adipose tissue [12].

In summary, we must remember that all items included in the reactive field region become part of the antenna. Thus, in the case of BAN, it is not possible to separate body and antenna.

\section{Preliminary Results}

The body being a loss dielectric interacting with the near field of the antenna, it will cause three main effects on the characteristics of the antenna: changing the antenna characteristics, reducing the heating performance by biological tissues and change in impedance.

The proposed antenna consists of a rectangular adapted loop (Fig.5), printed on an FR4_epoxy substrate $\left(\varepsilon_{\mathrm{r}}=4.4, \tan \delta=0.02\right.$ and $\left.\mathrm{h}=1.6 \mathrm{~mm}\right)$. Based on a parametric study, the loop is a conductor track with a width of $22 \mathrm{~mm}$ and a length of $30 \mathrm{~mm}$. The second loop has a width equal to $1 \mathrm{~mm}$ and a length of $17 \mathrm{~mm}$. His reflection coefficient $S_{11}$ is equal to $-17 \mathrm{~dB}$.
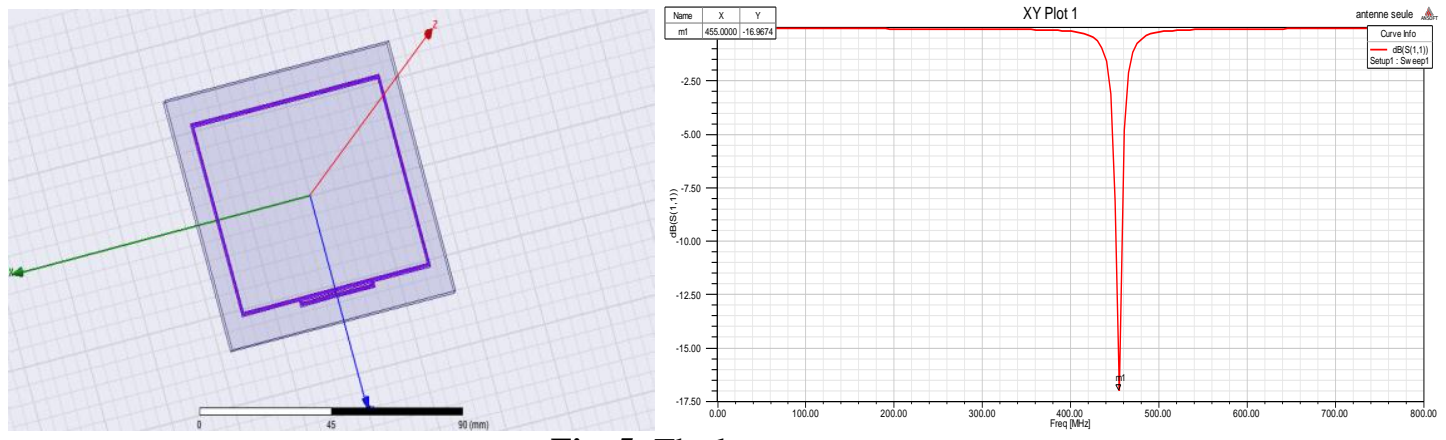

Fig. 5: The loop antenna

Our study will be made with a loop antenna disposed on a simplified body model of three-layer more exactly the thorax as shown in Fig.6 [13].

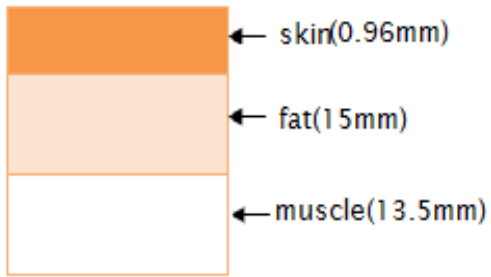

Fig. 6. Model of human thorax with thicknesses 
In this study, we will take into account two important parameters, firstly, the distance between the human body (in our case the thorax) and the antenna and secondly, the cardiac activity. The latter may be made by varying the thickness of the lowermost layer [1]. Indeed, the normal electrical conduction in the heart enables the pulse which is generated by the sinus node to spread. This stimulates the heart muscle (myocardium). Thus, it causes the contraction and relaxation of the muscle. This leads subsequently, variation of its thickness. Each layer is similar to a characteristic impedance line. The lowest layer (muscle) is considered as a layer with variable thickness. And the surface of the last (the skin) is regarded as a wave transmission medium. Therefore, the impedance of the muscle is changing as and as one progresses toward the surface. The lowermost layer ranges from $24 \mathrm{~mm}$ to $38 \mathrm{~mm}$ [14].

Fig.7 shows the characteristics of the loop antenna for $\mathrm{h}_{\text {muscle }}=38 \mathrm{~mm}$ for different distance of the human body (0mm, $5 \mathrm{~mm}$ and $10 \mathrm{~mm})$.
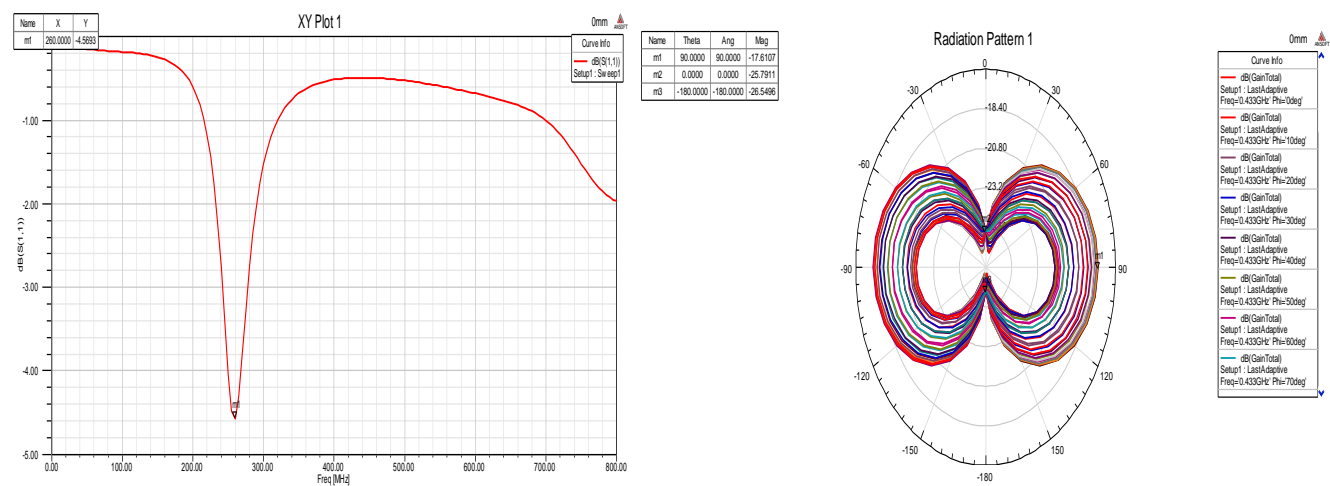

(a)
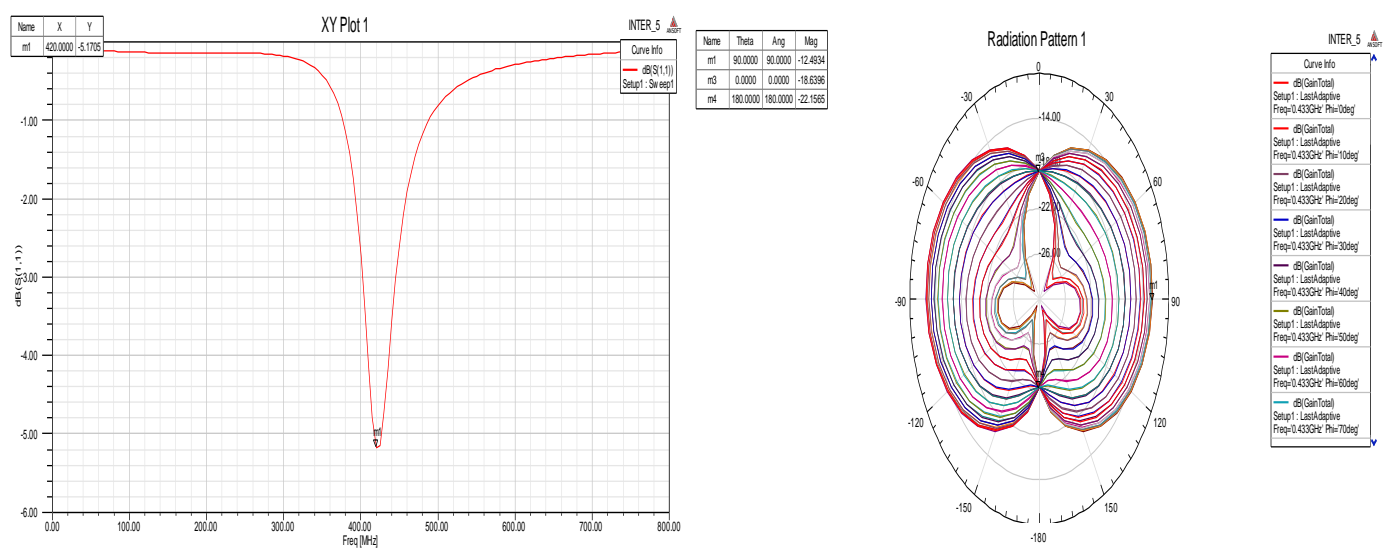

(b)
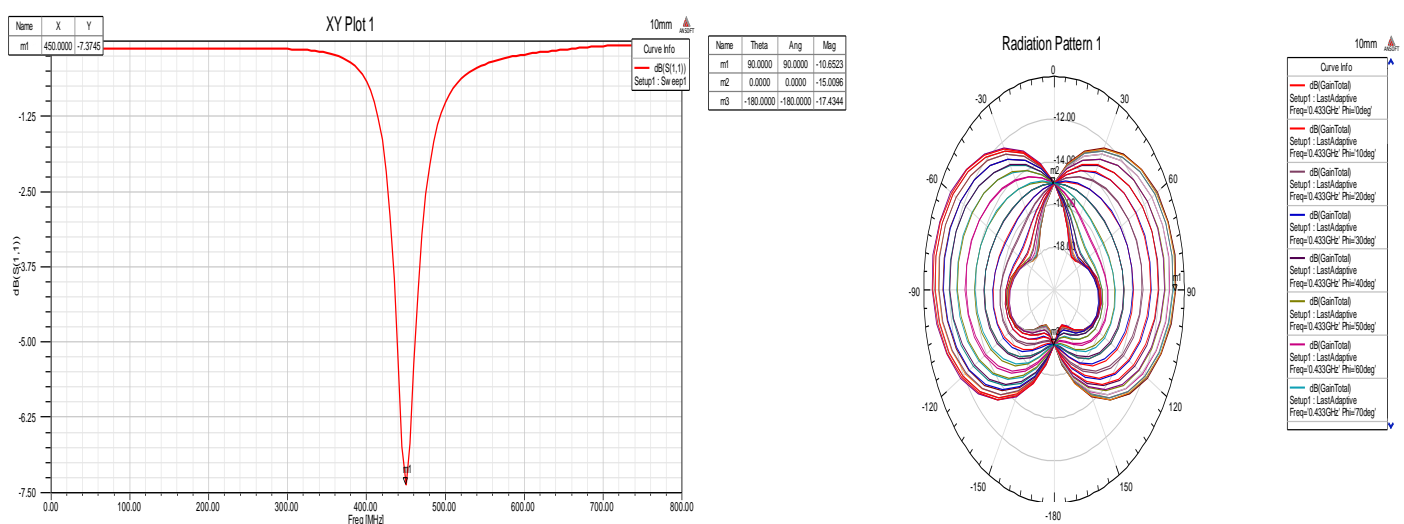

(c)

Fig.7.Antenna characteristics for $\mathrm{h}_{\text {muscle }}=38 \mathrm{~mm}$ for a distance from the body equal to: (a) $0 \mathrm{~mm}$, (b) $5 \mathrm{~mm}$ and (c) $10 \mathrm{~mm}$ 
From Fig.7, for a thickness of muscle equal to $38 \mathrm{~mm}$, the antenna resonance frequency, its reflection coefficient and its gain vary significantly depending on the distance from the body and also in relation to those of the single antenna (Fig.5). For a value of d equal to $10 \mathrm{~mm}$, the antenna resonates at $450 \mathrm{MHz}$ with a $S_{11}$ equal to $-7.3745 \mathrm{~dB}$ and a gain of $-10.65 \mathrm{~dB}$. Whereas for a distance of $0 \mathrm{~mm}$, the resonance frequency becomes equal to $260 \mathrm{MHz}$ with a $\mathrm{S}_{11}$ equal to $-4.56 \mathrm{~dB}$ while the gain is $-17.61 \mathrm{~dB}$.
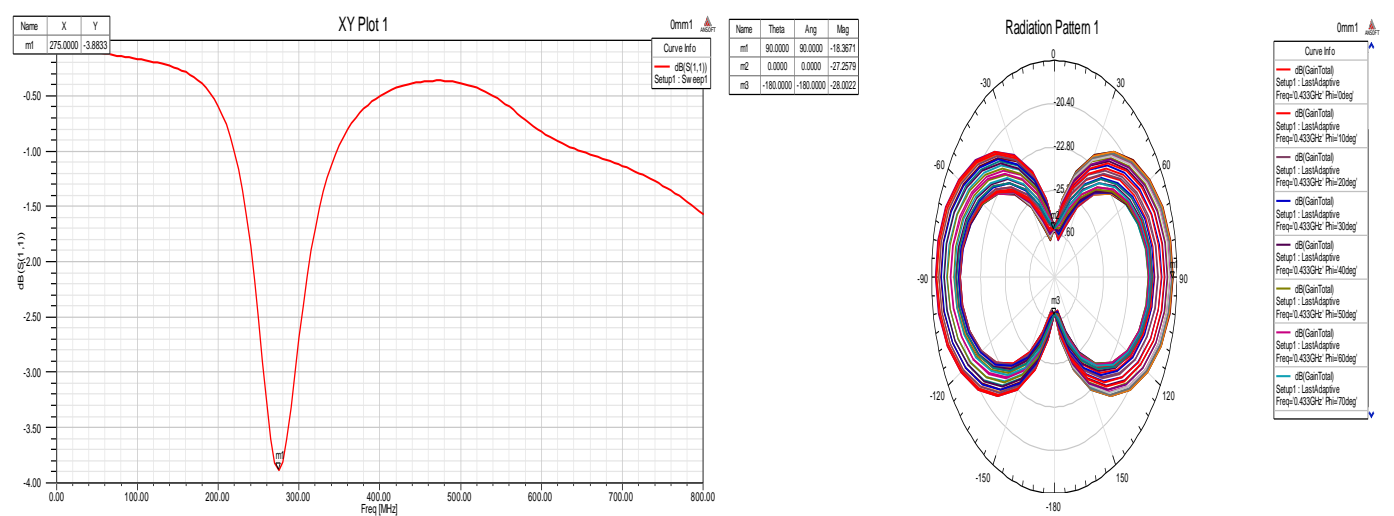

(a)
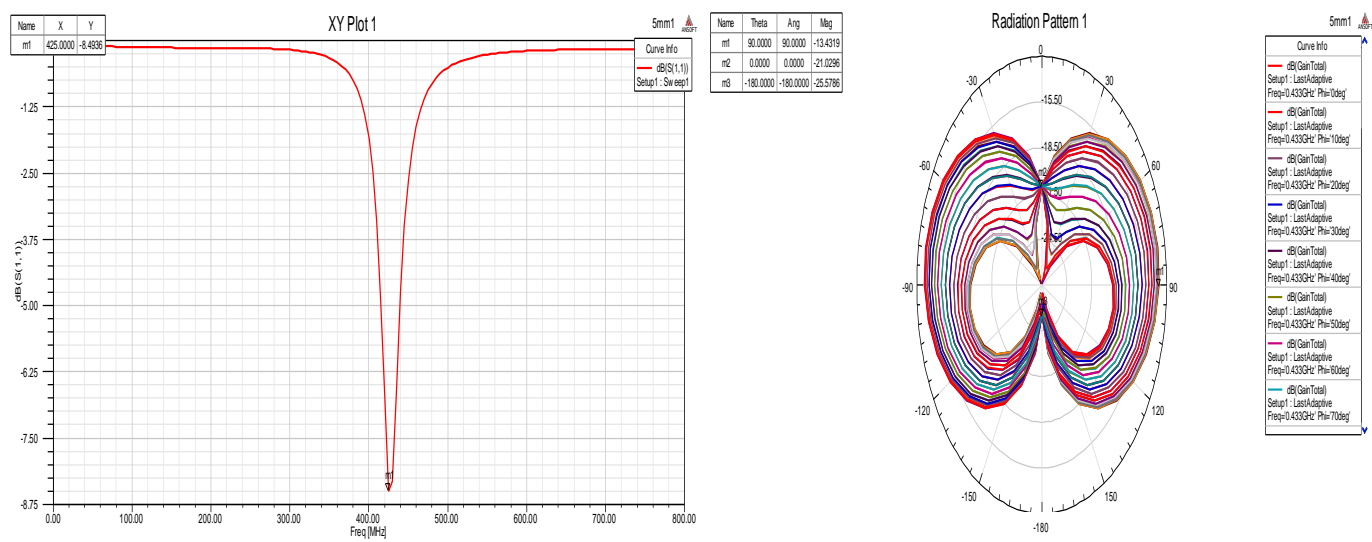

(b)
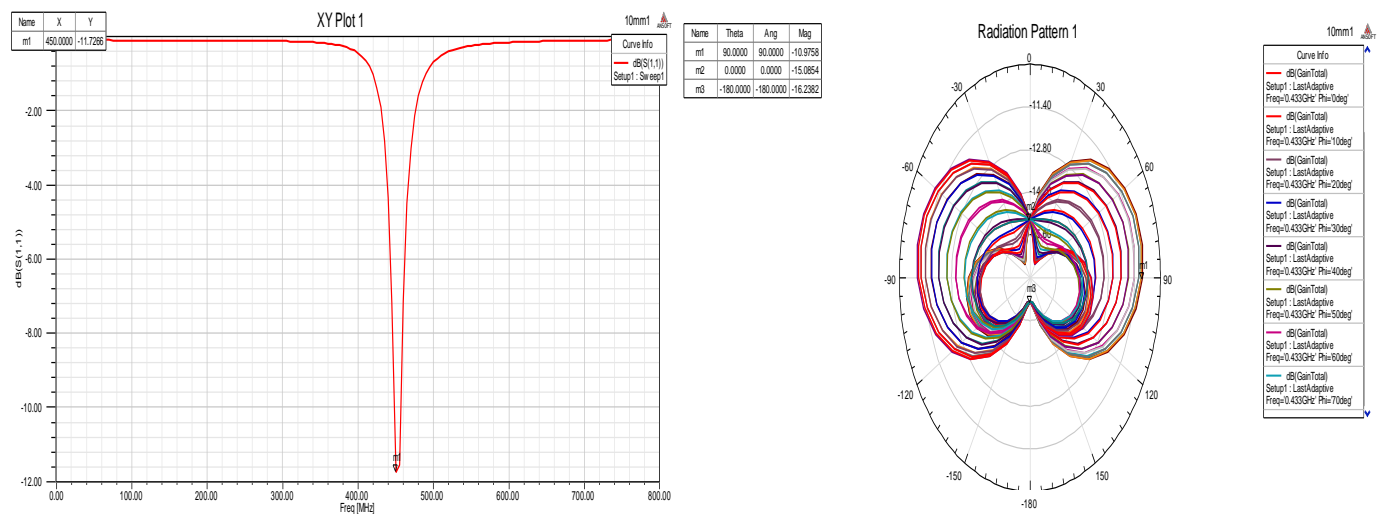

(c)

Fig.8. Antenna characteristics for $\mathrm{h}_{\text {muscle }}=24 \mathrm{~mm}$ for $\mathrm{d}$ equal to: (a) $0 \mathrm{~mm}$, (b) $5 \mathrm{~mm}$ and (c) $10 \mathrm{~mm}$

For $\mathrm{h}_{\text {muscle }}$ equal to $24 \mathrm{~mm}$, according to the Fig. 8 the antenna characteristics vary depending on the distance to the human body. If the antenna is placed directly on the thorax, its reflection coefficient is equal to $3.8 \mathrm{~dB}$ for a resonant frequency equal to $275 \mathrm{MHz}$ and a gain of $-18.367 \mathrm{~dB}$. This reduces, to a distance between the antenna and the body of $5 \mathrm{~mm}$, reaching $-13.43 \mathrm{~dB}$. While $S_{11}$ is equal to $-8.5 \mathrm{~dB}$ and it will be equal to $11.72 \mathrm{~dB}$ for $\mathrm{d}$ equal to $10 \mathrm{~mm}$. The results are summarized in Table 1. 
Table.1. Comparing antenna characteristics for different distance of the human body

\begin{tabular}{|l|l|l|l|l|}
\hline \multicolumn{2}{|l|}{ Distance from the human body } & Frequency & $\mathrm{S}_{11(\mathrm{~dB})}$ & $\mathrm{Gain}_{(\mathrm{dB})}$ \\
\hline \multirow{3}{*}{$0 \mathrm{~mm}$} & $\mathrm{~h}_{\text {muscle }}=24 \mathrm{~mm}$ & $275 \mathrm{MHz}$ & -3.8 & -18.367 \\
\cline { 2 - 5 } & $\mathrm{h}_{\text {muscle }}=38 \mathrm{~mm}$ & $260 \mathrm{MHz}$ & -4.5693 & -17.61 \\
\hline \multirow{2}{*}{$5 \mathrm{~mm}$} & $\mathrm{~h}_{\text {muscle }}=24 \mathrm{~mm}$ & $425 \mathrm{MHz}$ & -8.49 & -13.431 \\
\cline { 2 - 5 } & $\mathrm{h}_{\text {muscle }}=38 \mathrm{~mm}$ & $420 \mathrm{MHz}$ & -5.17 & -12.493 \\
\hline \multirow{2}{*}{$10 \mathrm{~mm}$} & $\mathrm{~h}_{\text {muscle }}=24 \mathrm{~mm}$ & $450 \mathrm{MHz}$ & -11.72 & -10.975 \\
\cline { 2 - 5 } & $\mathrm{h}_{\text {muscle }}=38 \mathrm{~mm}$ & $450 \mathrm{MHz}$ & -7.3745 & -10.652 \\
\hline
\end{tabular}

\section{Conclusion}

The human body is composed of a wide variety of biological medium with very different properties. The electromagnetic field passing through this medium depends on the thickness and the exact composition of biological tissues. The human body does not present an ideal environment for the propagation of RF waves, especially for high frequencies. This dissipative medium will reduce RF antenna performance especially in terms of efficiency and radiation pattern. The antenna is greatly disturbed when it is located near the body for distances less than $20 \mathrm{~mm}$, that is to say when the body is already in the sphere radians as explained in this paper. In this zone, the interaction is stronger. It will therefore be necessary to develop desensitization techniques. Insensibilisation means reduction of the body-antenna coupling and causing an increase in efficiency, less dependence on fat and possibly an increased radiation. Two techniques can be used: the reduction of the quality factor and the introduction of absorbing ferrite layers.

\section{References}

[1]. K.Tayari, M.Chaoui, H.Ghariani, M.Lahiani, Influence of the cardiac activity on the surface impedance of a multilayer medium, International conference on advanced technologies for signal and image processing, ATSIP'2014, Tunisia.

[2]. Y Kim, H Morishita, Y Koyanagi, K Fujimoto, A Folded Loop antenna system for handsets developed and based on the advanced design concept, IEICE Trans. Commum, Vol E84-B, No 9, September 2001.

[3]. J.V.Niekerk, F.L.Dacus, S.Bible, Loop Antenna Basics and Regulatory Compliance for Short-Range Radio, Microchip Technology Inc, 2002.

[4]. Y Saito, I Nagano, H Haruki, A novel Loop antenna for a wristwatch phone, IEICE Trans. Commum, Vol E84-B, No 5, May 2001.

[5]. Krupka Z., The Effect of the Human Body on Radiation Properties of Small-Sized Communication Systems, IEEE Transactions on Antennas Propagation, Vol. AP-16, No. 2, Mars 1968.

[6]. Andersen J. B., Balling P., Admittance and Radiation Efficiency of the Human Body in the Resonance Region, Proceedings of the IEEE, July 1972 .

[7]. Domenico Gaetano, Analysis and Design of Footwear Antennas, Dublin Institute of Technology, School of Electrical \& Electronic Engineering, September 2014.

[8]. Koichi Ogawa, Tomoki Uwano, and Masao Takahashi, A Shoulder-Mounted Planar Antenna for Mobile Radio Applications, IEEE TRANSACTIONS ON VEHICULAR TECHNOLOGY, Vol. 49, No. 3, MAY 2000.

[9]. T.Kellomaki, J. Heikkinen, M. Kivikoski, Wearable antennas for FM reception, Proc. European Conference on Antennas and propagation, April 2010.

[10]. King R. W. P., Transponder Antennas In and Near a Three-Layered Body, IEEE Transactions on Microwave Theory and Techniques, Vol. MTT-28, No. 6, Juin 1980.

[11]. Juho Poutanen, Interaction between mobile terminal antenna and user, HELSINKI UNIVERSITY OF TECHNOLOGY Department of Electrical and Communications Engineering Radio Laboratory, 2007.

[12]. Christ A., Klingenböck A., Samaras T., Goiceanu C., Kuster N., The Dependence of Electromagnetic Far-Field Absorption on Body Tissue Composition in the Frequency Range From $300 \mathrm{MHz}$ to $6 \mathrm{GHz}$, IEEE Transactions on Microwave Theory and Techniques, Vol. 54, No. 5, Mai 2006.

[13]. K.Tayari, H.Werfelli, M.Chaoui, H.Ghariani, M.Lahiani, Modeling of the thorax of human body at 433 MHz, ATSIP'2016, March 21-24, 2016, Tunisia.

[14]. kedar Nath Sahu, Challa Dhanunjaya Naidu, M. Satyam, and K. Jaya Sankar, Study of RF Signal Attenuation of Human Heart, Hindawi Publishing Corporation Journal of Engineering Volume 2015, Article ID 484686. 